\title{
Aborto terapéutico: ¿realmente existe?
}

\section{Therapeutic abortion: is there really?}

\section{Percy Pacora-Portella \\ 1. Médico ginecoobstetra. Departamento de Ginecología y Obstetricia, Universidad Nacional Mayor de San Marcos. Hospital Nacional Docente Madre-Niño San Bartolomé.}

\section{RESUMEN}

A propósito de la reciente promulgación de la Resolución Ministerial N..$^{\circ}$ 486-2014/Minsa, que aprueba la guía técnica nacional para el aborto terapéutico, se ha generado un gran debate al respecto. Como un aporte a la discusión, se presenta una revisión sobre las definiciones y tipos de aborto, para luego centrarse en el denominado 'aborto terapéutico' y explorar las dificultades que existen para definirlo. Finalmente, se presentan algunas observaciones a esta Guía técnica emitida por el Minsa. Se concluye que el término 'aborto terapéutico' es anacrónico y contradictorio porque el aborto no es un tratamiento que asegure mejorar la salud de la mujer ni la del niño. El término apropiado debería ser 'interrupción del embarazo por razones médicas'.

Palabras clave: aborto; aborto terapéutico; interrupción voluntaria del embarazo; salud humana.

\begin{abstract}
With regard to the recent enactment of the Peruvian Ministerial Resolution N. ${ }^{\circ}$ 486-2014/Minsa, which approves the national technical guide for therapeutic abortion, has generated considerable debate. A review of the definition and type of abortion and the focus on the so-called 'therapeutic abortion' is presented. This article explores the difficulties in defining it. Finally, some comments are presented to the Technical Guidance issued by the Minsa. We conclude that the term 'therapeutic abortion' is anachronistic and contradictory because abortion is not a treatment ensuring better health of the woman neither for the unborn child. The proper term should be 'termination of pregnancy for medical reasons'.
\end{abstract}

KErWORDS: abortion; therapeutic abortion; voluntary interruption of pregnancy; human health. 


\section{INTRODUCCIÓN}

Los médicos han llamado abortos a todas las pérdidas del embarazo antes de la semana 22 de gestación, mientras que a la muerte del niño dentro del útero a partir de la semana 22 la han denominado muerte fetal, óbito fetal o natimuerto. Sin embargo, los avances en biología reproductiva indican que esta clasificación es arbitraria, incompatible con el desarrollo embrionario y fetal y no es clínicamente útil. ${ }^{1}$ Un enfoque más útil es clasificar a la pérdida del embarazo en términos de etapas de desarrollo del niño durante la gestación.

Así, el período preembrionario dura desde la concepción hasta aproximadamente la semana 5 después del primer día del último período menstrual. El período embrionario comienza en la semana 6 de gestación y continúa hasta la semana 9. El período fetal comienza en la semana 10 de gestación, o 70 días a partir de la última menstruación, y se extiende a lo largo del embarazo hasta el parto. Así, desde el punto de vista de la biología del desarrollo, la pérdida del embarazo pueden ser clasificados como preembrionario (anembrionado), embrionario y fetal. ${ }^{1}$

\section{'ABORTO' VERSUS 'EL NIÑO MURIÓ ANTES DE LA SEMANA 22'}

El término aborto tiene su origen de la palabra latina abortus y en el idioma español tiene cuatro significados:2 1) acción de abortar; 2) interrupción del embarazo por causas naturales 0 deliberadamente provocadas, que eventualmente puede constituir un delito; 3) ser o cosa abortada; y 4) engendro, monstruo. ${ }^{2}$ De manera que al común de las personas -particularmente a los profesionales que cuidan la salud-, el término aborto tienen un significado peyorativo ('engendro', 'monstruo') y les trae a la memoria hechos con consecuencias desagradables. Por esta razón, es fácil de entender que en las historias clínicas no se investiguen los factores asociados a la muerte en las etapas preembrionaria, embrionaria y fetal del niño; y simplemente se les da a la mujer o a la pareja un listado de exámenes de laboratorio para que se lo realicen en forma ambulatoria y que regresen al consultorio por infertilidad. Naturalmente, esta práctica no ayuda a los profesionales de la salud ni a las parejas a comprender las causas que intervinieron en la pérdida del embarazo.

En cambio, si en lugar de decir 'ocurrió aborto en semana 6, 12 y 18 de gestación' dijéramos 'ocurrió la muerte de un niño en semana 6,12 y 18', la pregunta lógica después de esta afirmación sería ¿¿por qué se murió el niño?' Y en ese momento es cuando los profesionales de la salud debieran iniciar el proceso de identificación de los factores asociados a la muerte del niño antes de la semana 22, llamado usualmente aborto.

\section{¿QUÉ SIGNIFICA ABORTO DESDE EL PUNTO DE VISTA MÉDICO?}

Aborto significa la detención del desarrollo del niño antes de la semana 22 de gestación o la expulsión de un niño menor de 22 semanas de gestación. El código internacional es 003 . Por su naturaleza, los abortos pueden ser divididos en abortos espontáneos y abortos inducidos.

\section{Aborto espontáneo}

El proceso reproductivo humano aparece a simple vista como altamente ineficiente. Se ha llegado a calcular que hasta $65 \%$ de los embarazos humanos terminan con una pérdida subclínica. La incidencia del aborto clínico en la población general varía de $10 \%$ a $30 \%$, con un promedio de $15 \%$.

\section{Aborto inducido}

El aborto inducido es la interrupción de un embarazo por intervención humana. Existen cuatro tipos de abortos inducidos: libre, eugenésico, por razones terapéuticas y por motivaciones mixtas.

\section{Aborto libre}

Realizado bajo el supuesto derecho que tendría la mujer para interrumpir su embarazo. Las razones más frecuentes son las económicas y las sociales. Bajo este concepto, bastaría el hecho de ser un embarazo no deseado.

\section{Aborto eugenésico}

Pretende la eliminación de un feto cuando se puede predecir con probabilidad o certeza que nacerá con un defecto o enfermedad. 
Aborto por razones médicas o terapéuticas

Es aquella interrupción voluntaria de un embarazo antes de la viabilidad fetal por razones de salud materna.

\section{Aborto por motivaciones mixtas}

Se refiere a la reducción fetal selectiva, que pretende eliminar algunos embriones en el caso de embarazos múltiples, con el fin que otros tengan mejor probabilidad de sobrevivir.

\section{¿QUÉ ES EL ABORTO TERAPÉUTICO?}

El aborto terapéutico es la interrupción voluntaria de un embarazo antes de la viabilidad fetal (22 semanas o menos de $500 \mathrm{~g}$ ), por razones de salud materna. Sin embargo, la denominación de 'aborto terapéutico' no se ajusta a la realidad, ya que ningún aborto tiene la propiedad intrínseca de curar o mejorar el estado de salud de la mujer. En su lugar, debiera emplearse el término 'interrupción del embarazo por razones médicas'.

Si bien la Organización Mundial de la Salud (OMS) señala que el aborto inducido se realiza en condiciones seguras o inseguras, el hecho es que el aborto inducido realizado por ginecólogos entrenados, en la semana 8 de gestación, tiene una tasa de muerte materna de cerca de 1 por cada 100000 procedimientos.,4 El riesgo relativo de morir como consecuencia del aborto inducido se duplica aproximadamente por cada dos semanas después de la semana 8 de gestación. Los Centros para el Control y la Prevención de Enfermedades en EEUU registraron siete muertes maternas relacionadas con el aborto legal inducido en 2004. ${ }^{5}$ De manera que, contrario a lo que señala la OMS, no existe aborto seguro ya que muchas de estas muertes no son registradas. ${ }^{6}$

\section{Dificultades para definir el aborto terapéutico}

Existen cuatro preguntas que deben ser respondidas a la luz de nuestro conocimiento a fin de definir la finalidad del aborto terapéutico.

- Se justifica el aborto terapéutico en casos de embarazos que ponen en peligro la vida de la mujer. Entonces, la pregunta es: ¿qué embarazo pone en peligro la vida de la mujer?
- Se justifica el aborto cuando el embarazo agrava el pronóstico materno en caso de alguna enfermedad materna previa. Entonces, la pregunta es: ¿qué enfermedades se agravan durante el embarazo?

$\triangle$ Debido a que la OMS define a la salud como el estado de bienestar físico, mental y social y no solo la ausencia de enfermedad, si una mujer físicamente sana, solicita aborto porque esta mental o socialmente perturbada o enferma, la medicina debe ayudarla. Al aceptar tal definición todo aborto inducido sería terapéutico. Entonces, la pregunta es: ¿qué es salud?

^ Cualquier aborto provocado por un médico sería terapéutico, ya que los médicos realizan terapias. Entonces, la pregunta es: ¿qué es ser médico?

A continuación se responderá estas cuatro interrogantes.

¿Qué embarazos ponen en peligro la salud de la mujer?

Cualquier embarazo pone en peligro la salud de la madre y no por ello se deben interrumpir a todos los embarazos.

Al contrario, se le debe dar la seguridad física, psicológica, social y espiritual a toda gestante; y eso no se logra con una intervención quirúrgica, sino con una intervención médica que atienda a todas estas cuatro áreas del ser humano.

Así, la primera causa de muerte en Perú en las mujeres gestantes adolescentes es el suicidio. La primera causa de muerte en el embarazo en los hospitales del Perú es la preeclampsia y la primera causa de muerte en la sierra y la selva es la hemorragia puerperal.? De estas muertes maternas, $64 \%$ ocurren en mujeres que tuvieron atención prenatal y fueron catalogadas como 'embarazos de bajo riesgo'; $72 \%$, en mujeres con talla menor de $156 \mathrm{~cm} ; 42 \%$, en la sierra; $63 \%$ se asocian a la enfermedad vascular del embarazo (preeclampsia, eclampsia, desprendimiento prematuro de placenta) y $43 \%$, al nacimiento prematuro. $^{8}$ De manera que no existe embarazo que no lleve en sí el riesgo de muerte materna. $Y$ eso no significa que para evitar el riesgo de muerte materna, se deba eliminar a todos los embarazos porque llegaríamos al absurdo de eliminar a la humanidad, ya que la humanidad se desarrolla en el útero materno con el desarrollo del niño.,10 
¿Qué enfermedades de la mujer se agravan durante el embarazo?

En toda situación en que la mujer presente trastorno del ánimo o falta de ayuda social.10,11 Por lo tanto, se debe tratar el estado de ánimo de la mujer y crear redes sociales para su ayuda. No se justifica médicamente proceder a realizar operaciones innecesarias; por ejemplo, abortos 'terapéuticos' o 'sentimentales' porque la mujer está deprimida o ha sido víctima de violación. Así, como no está justificado realizar parto por cesárea en mujeres por pánico al dolor del parto.

En los hospitales de Lima, la causa más frecuente por la que se solicita la interrupción del embarazo por razones médicas es el cáncer avanzado que requiere tratamiento de quimioterapia 0 radioterapia para tratar la enfermedad de la madre. Sin embargo, los estudios observacionales de cánceres de mama, útero, ovario y otros tipos de cáncer en mujeres gestantes han demostrado que la postergación del tratamiento del cáncer en la madre no afecta el tiempo de sobrevida libre de enfermedad de la mujer. Además, el embarazo no empeora la historia natural del cáncer. Debido a que el nacimiento prematuro se asocia fuertemente con efectos adversos, el permitir el nacimiento del niño a término es de capital importancia en mujeres gestantes con cáncer. ${ }^{12-18}$

Debido a que el cáncer de la madre en el embarazo se diagnostica en etapas tempranas, la quimioterapia y la cirugía radical deben evitarse en el primer trimestre del embarazo debido a la teratogénesis y la alta frecuencia de abortar. La cirugía que preserva el embarazo seguido de quimioterapia en el segundo o tercer trimestre, el parto a término y la quimioterapia neoadyuvante con subsecuente cirugía que complete el tratamiento parecen opciones de tratamiento viable. Todo cáncer en el embarazo necesita ser abordado en forma individual. ${ }^{12-18}$

La mortalidad materna relacionada con el embarazo en mujeres con cardiomiopatía hipertrófica es baja y parece estar confinada a las mujeres con un perfil de alto riesgo antes del embarazo. La mortalidad fetal es comparable a la de la población general; sin embargo, el riesgo de parto prematuro esta aumentado. ${ }^{19}$
¿Qué es la salud?

Al contrario de lo que dice la OMS, la salud no es un estado de completo bienestar físico, mental y social. La salud es un estado de equilibrio armónico entre el organismo humano en sus cuatro áreas físico, social, psicológica y espiritual, con el medio ambiente. Equilibrio armónico significa que la persona mantiene la homeostasis porque tiene paz interior y recibe ayuda o cooperación social.11,20,21

Numerosas condiciones que causan angustia o dolor incapacitante o socialmente incapacitante no califican para el aborto terapéutico.

\section{¿Qué es ser médico?}

El médico es una persona humana que tiene el conocimiento de cuidar y el arte de conservar la salud. La medicina, como profesión, es la aplicación del conocimiento del cuidado de la salud y el arte de conservar la fe y la esperanza de las personas en la vida para que la vida perdure siempre. El que esto ocurra depende de la población que observa el accionar del profesional de la medicina y desarrolle el poder curativo mediante la fe en la vida. ${ }^{22}$ En esencia, un médico es una persona buena que desea hacer el bien sin mirar a quien actuando con sabiduría. Sabiduría significa emplear el conocimiento para hacer el bien.

\section{OBSERVACIONES A \\ LA GUIIA TÉCNICA NACIONAL}

Las observaciones a la Guía Técnica Nacional para la Estandarización del Procedimiento de la Atención Integral de la Gestante en la Interrupción Voluntaria por Indicación Terapéutica del Embarazo Menor de 22 semanas con Consentimiento Informado en el Marco de lo Dispuesto en el Artículo $119^{\circ}$ del Código Penal. ${ }^{23}$

$\Delta$ Las primeras dos indicaciones de esta guía señalan como indicación al embarazo ectópico y a la enfermedad del trofoblasto (enfermedad molar). Todo médico especialista en ginecología y obstetricia sabe que no se requiere realizar junta médica para tratar el embarazo ectópico ni la enfermedad del trofoblasto. Todo médico sabe que estos embarazos, al comprometer la salud de la mujer, debe ser interrumpido por razones médicas. 
^ Los estudios reportados actualmente señalan que actualmente no hay justificación para interrumpir embarazos por razones de cáncer ginecológico que es una de las principales razones que antes se argüían para interrumpir el embarazo. El embarazo no altera la historia natural del cáncer del cáncer en la madre durante el embarazo como antes se creía.

- Todo médico sabe que las enfermedades crónicas avanzadas con grave daños de varios sistemas requiere un tratamiento individual y que más que interrumpir un embarazo, el cual es un procedimiento quirúrgico, requiere que el médico, la familia y la comunidad le brinden el apoyo psicológico, social y espiritual para mantener la homeostasis y lograr la recuperación. Esto, por supuesto, no se logra con la interrupción del embarazo.

- Esta guía técnica no considera que el médico siempre debe dar buenas noticias y no debe dar pronósticos basados en estadísticas porque las estadísticas solo cuentan historias pasadas y no fenómenos vivos que están sucediendo en la actualidad.

- La interrupción médica del embarazo solo se justifica en casos poco frecuentes, por lo que deben ser evaluados mediante una junta médica y no requiere una norma técnica como la que ha publicado el Ministerio de Salud.

- Esta guía es una puerta de entrada para introducir legalmente el 'aborto eugenésico' y 'el aborto del embarazo no planeado o deseado', debido a que la madre esta perturbada psicológicamente o ha sido víctima de abuso sexual.

\section{CONCLUSIONES}

$\Delta$ El aborto o la pérdida del niño tempranamente es determinado por la herencia y el medio ambiente.

- La pérdida del niño tempranamente es consecuencia de la participación aislada 0 simultánea de factores patógenos estresores de naturaleza tóxico-contaminante, psicológico, social, nutricional, infeccioso, anatómico, vascular y metabólico.

- Debido a que toda intervención médica lleva riesgo a la salud humana, ninguna interrupción del embarazo médico o quirúrgico es seguro. La mejor cirugía es la que no se realiza.

$\Delta$ El término 'aborto terapéutico' es anacrónico y contradictorio porque el aborto no es un tratamiento que asegure mejorar la salud de la mujer ni la del niño. El término apropiado debería ser 'interrupción del embarazo por razones médicas'.

- El médico es una persona que busca la verdad y defiende la vida, empleando el conocimiento para hacer el bien, es decir, actuando con sabiduría.

- La vida empieza con las palabras de amor y esperanza que ingresan a través del oído, particularmente al niño que se desarrolla en el cuerpo de la madre.

\section{REFERENCIAS BIBLIOGRÁFICAS}

I. Silver RM, Branch DW. Sporadic and recurrent pregnancy loss. In: Reece EA, Hobbins JC, Gant NF, Silver RM, Branch DW, editors. Handbook of Clinical Obstetrics: The Fetus \& Mother. 3th ed. Malden, Massachusetts, USA: Blackwell; 2007, p. I43-160.

2. Real Academia Española. Diccionario de la lengua española. $22^{\mathrm{a}}$ ed. Madrid: Espasa, 200I.

3. Grimes DA. Estimation of pregnancy-related mortality risk by pregnancy outcome, United States, 1991 to 1999. Am J Obstet Gynecol. 2006; 194(I):92-94.

4. Strauss LT, Gamble SB, Parker WY, Cook DA, Zane SB, Hamdan $S$, Centers for Disease Control and Prevention (CDC).Abortion Surveillance- United States, 2004. MMWR Surveill Summ. 2007;56(SS-9): I-33.
5. Gamble SB, Strauss LT, Parker WY, Cook DA, Zane SB, Hamdan $S$, Centers for Disease Control and Prevention (CDC).Abortion Surveillance- United States, 2005. MMWR Surveill Summ. 2008;57(SS-13): I-32.

6. Horon IL. Underreporting of maternal deaths on death certificates and the magnitude of the problem of maternal mortality. Am J Public Health. 2005;95(3):478-482.

7. Del Carpio-Ancaya L. Situación de la mortalidad materna en el Perú, 2000-2012. Rev Peru Med Exp Salud Publica. 20I3;30(3):46I-464.

8. Pacora P, Aguirre V, Gonzales G. Factores condicionantes de la muerte materna en los hospitales del Ministerio de Salud del Perú 2000-2010. De próxima aparición 2014. 
9. Pacora P.Ética en la atención de la salud de la mujer. Diagnóstico (Perú) 201 I;50(3): 144-150 [citado 20 de setiembre 2014]. Disponible en: http://www.fihu-diagnostico.org.pe/revista/ numeros/20I I/jul-set//44-I50.html

10. Pacora P, Gonzales G, Perez Y.Atención del embarazo temprano. Diagnóstico (Perú) 2013;52(2):6I-73.

II. Pacora P. Factores determinantes de la salud y condicionantes de la enfermedad en la mujer peruana. Diagnóstico (Perú) 2012;5I(3):I25-133.

12. Monleon J, Goberna L, Monleon FJ. Cáncer y gestación. Clin Invest Gin Obst. 2006;33(3):80-92

13. Dobashi M, Isonishi S, Morikawa A,Takahashi K, Ueda K, Umezawa $\mathrm{S}$, et al. Ovarian cancer complicated by pregnancy:Analysis of 10 cases. Oncol Lett. 2012;3(3):577-580.

14. Machado F, Vegas C, Leon J, Perez A, Sanchez R, Parrilla JJ, et al. Ovarian cancer during pregnancy: analysis of 15 cases. Gynecol Oncol. 2007; 105(2):446-450.

15. Ferraioli D, Buenerd A, Marchiolè P, Constantini S, Venturini PL, Mathevet P. Early invasive cervical cancer during pregnancy: different therapeutic options to preserve fertility. Int J Gynecol Cancer. 20I2;22(5):842-849.

16. Guo Q, Shan Y, Yang JX, Liu JT, Cao DY, Cheng NH, Huang HF, Pan LY, Lang JH, Shen K. [Management of invasive cervical cancer in pregnancy: clinical analysis of 13 cases]. [Article in Chinese] Zhonghua Fu Chan Ke Za Zhi. 20I2;47(I2):893-897.

17. Germann N, Haie-Meder C, Morice P, Lhomme C, Duvillard P, Hacene K, et al. Management and clinical outcomes of pregnant patients with invasive cervical cancer.Ann Oncol. 2005; 16(3):397402.
18. Loibl S, Han SN, von Minckwitz G, Bontenbal M, Ring A, Giermek J, et al. Treatment of breast cancer during pregnancy: an observational study. Lancet Oncol. 2012; I3(9):887-896.

19. Schinkel AF. Pregnancy in women with hypertrophic cardiomyopathy. Cardiol Rev. 20।4;22(5):217-222.

20. Pacora P, Oliveros M, Kendall R, Ticona M, Huanco D. Factores determinantes de la salud, la enfermedad y la muerte en el niño peruano. En: Pacora P, Oliveros M, Kendall R, Guibovich A, Calle $M$, Cano U, Cueva G, editores. Atención primaria de la mujer, la niñez y la adolescencia en el Perú. Lima: Perú Saludable; 2009. p. 43-62.

21. Pacora P. Factores determinantes de la salud y condicionantes de la enfermedad. En: Avila D, Fescina R, Romero R, editores. Obstetricia y Perinatología. Libro homenaje al Prof. Dr. Samuel Karchmer. Guayaquil, Ecuador: Federación Latinoamericana de Asociaciones de Medicina Perinatal; 2013. p. 445-453.

22. Pacora P. Ser médico: La esencia del médico y su práctica. En: Pacora P, Oliveros M, Kendall R, Guibovich A, Calle M, Cano $U$, Cueva G, editores. Atención primaria de la mujer, la niñez y la adolescencia en el Perú. Lima: Perú Saludable; 2009. p. |91212 [citado 20 setiembre 20l4]. Disponible en: http://www. perusaludable.org/CAPITULO\%207.pdf

23. República del Perú. Ministerio de Salud (MINSA). Resolución Ministerial No 486-20I4/MINSA, del 27 de junio del 20I4, que aprueba la Guía Técnica Nacional para la estandarización del procedimiento de la Atención Integral de la gestante en la Interrupción Voluntaria por Indicación Terapéutica del Embarazo menor de 22 semanas con consentimiento informado en el marco de lo dispuesto en el artículo $119^{\circ}$ del Código Penal.

\section{Correspondencia}

Dr. Percy Pacora-Portella

ppacorap@gmail.com

Fecha de recepción: 2 de octubre de 2014

Fecha de aceptación: 6 de octubre de 2014

\section{Conflictos de interés}

El autor declara no tener conflictos de interés durante el planteamiento, ejecución de la investigación y la elaboración del artículo para su publicación. 